\title{
Description Logics Approach to Semantic Matching of Web Services
}

\author{
S. Colucci ${ }^{1}$, T. Di Noia ${ }^{1}$, E. Di Sciascio ${ }^{1}$, F. M. Donini ${ }^{2}$ and M. Mongiello ${ }^{1}$ \\ ${ }^{1}$ Politecnico di Bari, Italy \\ ${ }^{2}$ Universitá della Tuscia, Viterbo, Italy
}

\begin{abstract}
As more resources and services become available on the Web, there is a growing need for infrastructures that, based on advertised descriptions, semantically match in a peer-to-peer way providers with requesters of web services. We address the problem of matchmaking of web services from a knowledge representation perspective. Based on our approach we propose match categorization in terms of exact match, potential match - when request and offer though not identical are compatible - and partial match - when one or more inconsistency is present and rank of matches within categories. Then we report on our implementation of the proposed matchmaking framework in a prototype system.
\end{abstract}

Keywords: knowledge representation, semantic web, facilitator, services.

\section{Introduction}

The advent of the Internet and of its most noticeable offspring, the World Wide Web, has initially led to an explosion of information available on-line. The lack of infrastructures to help in accurately locate and keep up to date the huge amount of information and resources at hand, has resulted, over a few years, in an information overload, basically because of the inherent unstructured nature of web data.

The Semantic Web [1] initiative has begun, slowly yet steadily, to revolutionize the way information is provided on the Internet. The basic idea is to structure information with the aid of markup languages, based on the XML language, such as RDF [21] and DAML+OIL [18]. These languages have been conceived to allow for representation of machine understandable, unambiguous, description of web content through the creation of arbitrary domain ontologies, and aim at increasing openness and interoperability in the web environment. Building on the Semantic Web foundations, also a flourishing of languages for the description of web services has been recently witnessed, struggling to become accepted de-facto standards, such as WSDL [8] and DAML-S [24].

Widespread availability of resources and services enables - among other advantages - the interaction with a number of potential counterparts. The bottleneck is that it is difficult to find matches, possibly the best ones, between parties. Matchmaking is the process of searching the space of possible matches between demands and supplies, finding the best available ones[12, $20,13,23,25,24,26,14,19,27,16,7,9]$. Demands and supplies are here meant to represent web services, information, tangible or intangible goods, practically finding for any request an appropriate offer, or vice versa. Matchmaking is quite different from simply finding, given a demand a perfectly matching supply (or vice versa). Instead, it includes finding all those supplies that can to some extent fulfill a demand, and eventually propose the best ones. So the scenario, which is basically envisaged by the Semantic Web initiative, is one where peer entities may propose their goods and services and dynamically deal with counteroffers or further specifications, through the mediation of a matchmaking infrastructure.

A matchmaking infrastructure should then receive and store advertisement descriptions by both demanders and suppliers, and, as dynamically new demands or supplies are submitted, find most satisfying matches and return them. 


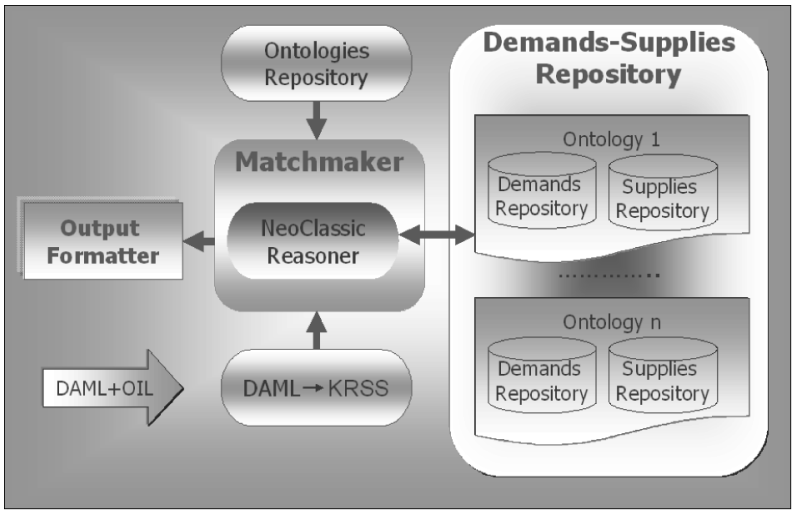

Fig. 1. The architecture of the engine.

The infrastructure has to treat in a uniform way suppliers and demanders, and base the matches on common, extensible ontologies for describing both supplies and demands [11].

Knowledge representation - in particular Description Logics (DL) - can deal with a uniform treatment of knowledge from suppliers and demanders, by modelling both as generic concepts to be matched.

In fact, the logical approach - which DLS are based upon - allows for an open-world assumption. Incomplete information is allowed (and can be filled after a selection of possible matches), and absence of information can be distinguished from negative information, allowing to discard offers/requests without the necessary properties, and to ask for missing information in the potential matches. The importance of ranking cannot be underestimated, as it is of extreme importance for practical use of the approach. The key questions that have to be answered in a dynamic framework are how far is a given demand (supply) from a potential counterpart? And which are the requirements that would eventually fulfill it? Such questions have to be answered relying on publicly available algorithms, to enforce trust and prevent rising of doubts on fairness of the proposals returned by the matchmaking facilitator.

In this paper we will concentrate on descriptions, regardless of the good or service considered. Although the logical framework is basically independent from the application adopted, we strictly relate our description to CLASSIC $[3,4]$. CLASSIC is a knowledge representation system that, although not endowed of a language expressive as more recent reasoners, e.g.,

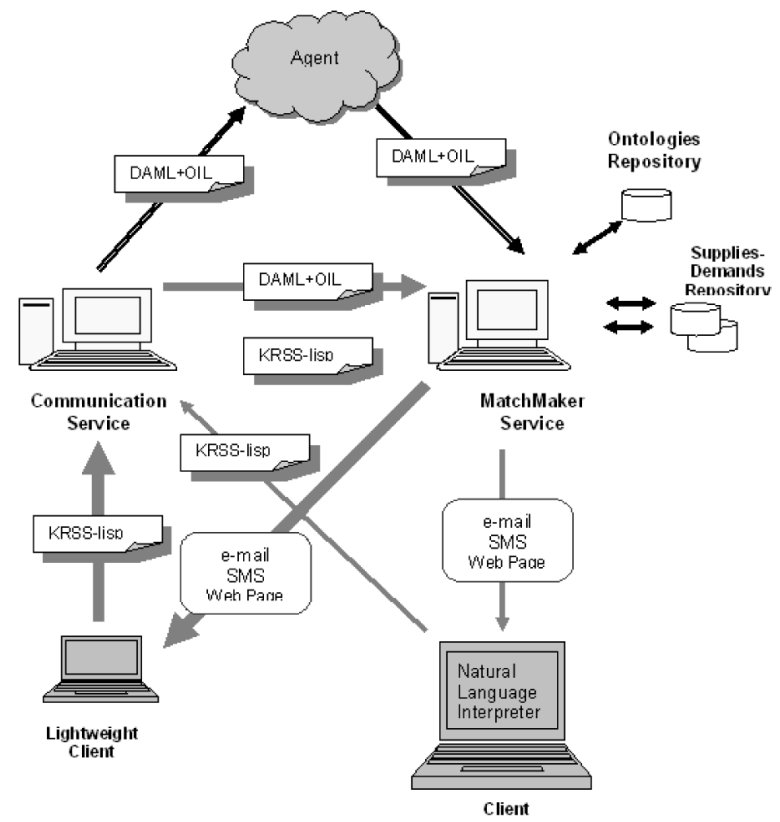

Fig. 2. The architecture of the matchmaking infrastructure.

FaCT [17] and Racer [15], has polynomial-time inferences and, most important, is a real system, endowed of concrete datatypes and can be simply wrapped into a host system.

\section{Description Logics basics}

DLs $[2,10]$ are a family of logic formalisms whose basic syntax elements are concept names, e.g., person, degree, and role names, such as workingIn, requiredAS. Intuitively, concepts stand for sets of objects, and roles link objects in different concepts. Formally, concepts are interpreted as subsets of a domain of interpretation $\Delta$, and roles as binary relations (subsets of $\Delta \times \Delta)$. Basic elements can be combined using constructors to form concept and role expressions, and each DL has its distinguished set of constructors. Every DL allows one to form a conjunction of concepts, usually denoted as $\sqcap$; some DL include also disjunction $\sqcup$ and complement $\neg$ to close concept expressions under boolean operations. Roles can be combined with concepts using existential role quantification, e.g., Graduate $\sqcap$ Эhas Degree.Engineering, which describes the set of graduates with an engineering degree, and universal role quantification, e.g., Person $\square$ $\forall$ livingIn.Apulia, which describes persons living exclusively in Apulia. Other constructs 
may involve counting, as number restrictions: Person $\Pi(\leq 1$ Degree $)$ expresses persons with at most one degree, and Person $\Pi(\geq 3$ Specialization) describes persons endowed of at least three specializations. Many other constructs can be defined, increasing the expressive power of the DL, up to n-ary relations [6]. Concept expressions can be used in inclusion assertions, and definitions, which impose restrictions on possible interpretations according to the knowledge elicited for a given domain. For example, we could impose that faculties can be divided into scientific and art ones, using the two inclusions Faculty $\sqsubseteq$ Scientific $\sqcup$ SocialSciences and Scientific $\sqsubseteq \neg$ Social Sciences. Or that graduates have at least one degree as Graduate $\sqsubseteq(\geq 1$ hasDegree $)$. Historically, sets of such inclusions are called TBox (Terminological Box). The basic reasoning problems for concepts in a DL are satisfiability, which accounts for the internal coherency of the description of a concept (no contradictory properties are present), and subsumption, which accounts for the more general/more specific relation among concepts, that forms the basis of a taxonomy. More formally, a concept $\mathrm{C}$ is satisfiable if there exists an interpretation in which $\mathrm{C}$ is mapped into a nonempty set, unsatisfiable otherwise. If a TBox $\mathrm{T}$ is present, satisfiability is relative to the models of $\mathrm{T}$, that is, the interpretation assigning $\mathrm{C}$ to a nonempty set must be a model of the inclusions in T. For instance, the concept AFaculty $\sqsubset$ Scientific $\Pi$ SocialSciences is clearly unsatisfiable w.r.t. the TBox containing the inclusion scientific and social sciences. A concept $\mathrm{C}$ subsumes a concept $\mathrm{D}$ if every interpretation assigns to $\mathrm{C}$ a subset of the set assigned to $\mathrm{D}$. Also subsumption is usually established relative to a TBox, a relation that we denote $T=C D$. Also, a TBox can be considered satisfiable if there exists at least one model (i.e., an interpretation fulfilling all its inclusions in a nontrivial way). It is important to note that in the CLASSIC system we use, each $C$ concept has an equivalent normal form as $C_{\text {names }} \sqcap C_{\sharp} \sqcap C_{\text {all }}$, in which $C_{\text {names }}$ is a conjunction of names, $C_{\sharp}$ of number restrictions, and $C_{\text {all }}$ of universal role quantifications. In the normal form, also all inclusions, definitions and disjoint groups have been made explicit [4]. CLASSIC provides the two basic reasoning services of DL-based systems, namely Concept Satisfiability (given a TBox $T$ and a concept $C$, does there exist at least one model of $T$ assigning a non-empty extension to $C$ ?), and Subsumption (given a TBox $T$ and two concepts $C$ and $D$, is $C$ more general than $D$ in any model of $T$ ?). Being a complete KR system, CLASSIC provides also data types as numbers and strings, and other services which are useful in a deployed prototype.

\section{Principles for matchmaking}

We highlight here common sense principles that a semantics-based approach should yield. First of all, a matchmaking facilitator of practical use has to be liberal enough about details, without preventing a proposer to fill in forms with (say) 30 or more different characteristics to be set. This implies that the absence of a characteristic in the description of a requested or offered profile should not be interpreted as a constraint of absence. Instead, it should be considered as a characteristic that could be either refined later, or left open if it is irrelevant for a user - which is called open-world assumption in KR. Obviously, the algorithm employed for matching should also take this issue into account. Secondly, a matchmaking system may give different evaluations, depending on whether it is trying to match a request $S$ with an offer $D$, or $D$ with $S$ - i.e. depending on who is going to use this evaluation. This requirement is already evident when characteristics are modelled as sets of words. Of course, using sets of words to model supplies and demands would be too sensible to the choice of words employed - it misses meanings related by words. It is now a common opinion that, it terms have a logical meaning, such fixed-terminology problems are overcome through an ontology [11]. Hence, we assume that supplies and demands are expressed in a DL. Obviously, this approach includes the sets-of-keywords, since a set of keywords can be considered also as a conjunction of concept names. We assume also that the common ontology is established as a TBox in $D L$. Now a match between a supply $S$ and a demand $D$ could be evaluated according to $T$. Let $T \models \ldots$ denote logical implication (truth in all models of $T$ ), and let $\sqsubseteq$ (subsumption) denote also implication between constraints of $S$ and $D$. There are three relations between concepts that we consider meaningful in semantic matchmaking: 
Implication. If $T \models(D \sqsubseteq S)$, then every constraint imposed by $D$ is fulfilled (implied) by $S$, and vice versa if $T \models(S \sqsubseteq D)$. This relation extends the previous set-based inclusion to general concepts. If both $T \models(D \sqsubseteq S)$ and $T \models(S \sqsubseteq D)$, then $D$ and $S$ should be considered equivalent in $T$. This relation extends exact matching by ruling out irrelevant syntactic differences. Consistency. If $D \sqcap S$ is satisfiable in $T$, then there is a potential match, in the sense that the constraints of neither proposal exclude each other. This relation has also been highlighted by other researchers [26]. However, that proposal lacks a ranking between different potential matches, which we believe is fundamental in order to support e.g., a project manager in the choice of the most interesting curricula, among all potential ones. Inconsistency. Otherwise, if $D \sqcap S$ is unsatisfiable in $T$, some constraints of one proposal are in contrast with the properties of the other one. However, also (say) supplies which are inconsistent with $D$ may be reconsidered if the demander accepts to revise some of $D$ 's constraints. We call this situation a near miss or partial match. The point, of course, is in not revising too much. Hence, also in this case a ranking - different from the one of potential matches - is fundamental.

We now state some properties that - we believe - every ranking function should have in logical matchmaking. First of all, a ranking for semantic matchmaking should be syntax-independent. That is, for every pair of supplies $S_{1}$ and $S_{2}$, demand $D$ and ontology $T$, when $S_{1}$ is logically equivalent to $S_{2}$ then $S_{1}$ and $S_{2}$ should have the same ranking for $D-$ and the same should hold also for every pair of logically equivalent demands $D_{1}, D_{2}$ with respect to every supply $S$. Secondly, a ranking for semantic matchmaking should be monotonic over subsumption. That is, for every demand $D$, for every pair of supplies $S_{1}$ and $S_{2}$, and ontology $T$, if $S_{1}$ and $S_{2}$ are both potential matches for $D$, and $T \models\left(S_{2} \sqsubseteq S_{1}\right)$, then $S_{2}$ should be ranked either the same, or better than $S_{1}$. The same should hold also for every pair of demands $D_{1}, D_{2}$ with respect to a supply $S$. Intuitively, this property could be read as "A ranking of potential matches is monotonic over subsumption if the more specific, the better." When turning to partial matches, adding another characteristic to an unsatisfactory proposal may either worsen its ranking (when another characteristic is violated) or keep it the same (when the new characteristic is not in contrast). Note that this ranking should be kept different from the ranking for potential matches. Obviously, the properties pointed out here are independent of the particular DL employed, or even the particular logic chosen. For instance, the same properties could be stated if propositional logic was used or also logics, such as DAML, for which reasoning systems are still unavailable.

\section{Finding best matches}

Here we present algorithms for matchmaking, which satisfy previously stated properties and have been devised adapting the original CLASSIC structural algorithm for subsumption [4]. Only the algorithm for potential match is completely outlined; the partial match is similar, and is only briefly described here due to a lack of space. Recall that a CLASSIC concept $C$ can be put in normal form as $C_{\text {names }} \sqcap C_{\sharp} \sqcap C_{\text {all }}$. Without ambiguity, we use the three components also as sets of the conjoined concepts. Moreover, recall that the TBox in CLASSIC can be embedded into the concepts, hence we do not consider explicitly the TBox, although it is present.

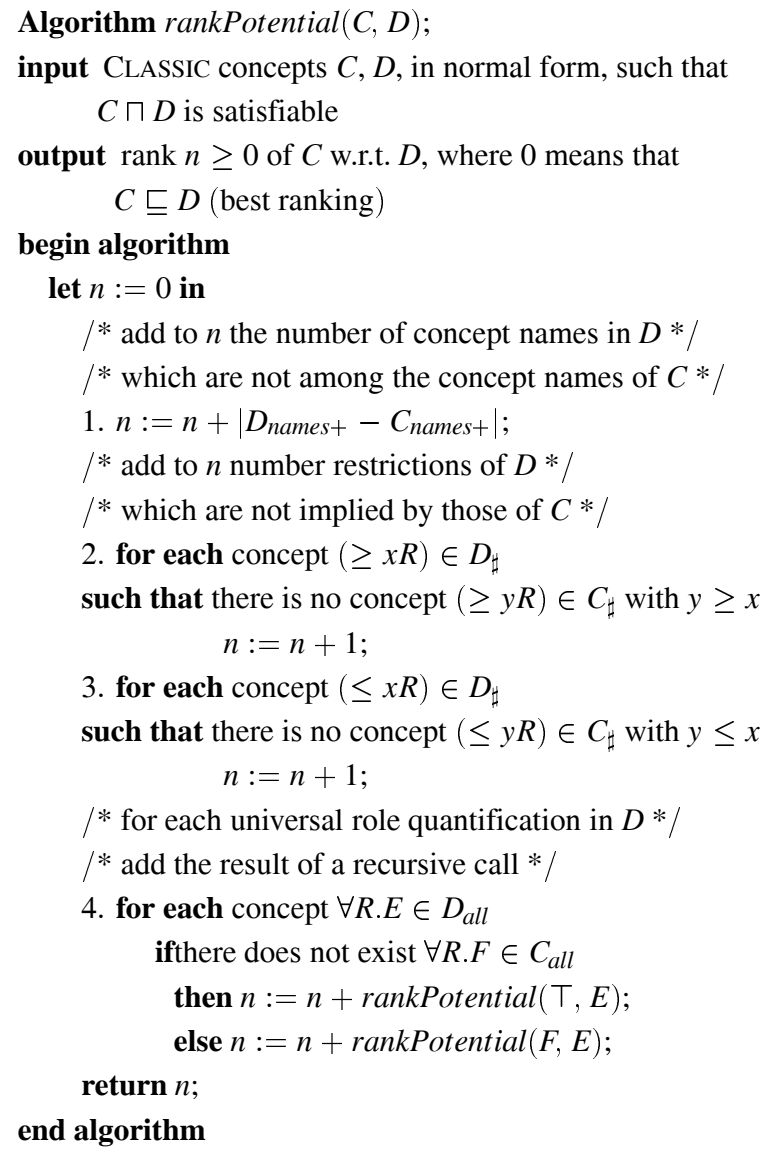


Obviously, total match is a particular case of potential match, obtained when rankPotential $(C$, $D)=0$. With reference to the complexity, which is extremely important for practical use of the system, the expansion of the TBox in the construction of the normal form can lead to an exponential blow-up, as demonstrated by Nebel in [22]. Anyway, a polynomial algorithm cannot be expected since subsumption in $\mathcal{A L}$ with an acyclic TBox $\mathcal{T}$ is co-NP-hard [5]. However, in the cited paper Nebel argues that the expansion is exponential in the depth of the hierarchy $\mathcal{T}$; if the depth of $\mathcal{T}$ is $O(\log |\mathcal{T}|)$, then the expansion is polynomial, and so is the above algorithm. Notice that the algorithm penalizes generic profile descriptions, which in simple subsumption matching would be unfairly advantaged.

The algorithm for ranking partial matches follows again the partition of CLASSIC concepts into names, number restrictions, and universal role quantifications. However, this time we are looking for inconsistencies. Hence, when a universal role quantification is missing in either concept, the recursive call is unnecessary. For both algorithms it can be proved they respect the properties highlighted in the previous section.

\section{A protoype system}

The matchmaking framework presented in the previous sections has been deployed in a prototype facilitator. The system embeds a NeoClassic engine $(\mathrm{a} \mathrm{C}++$ implementation of the original CLASSIC system, whose sources have been adapted for our purposes).

The general architecture of the system is shown in Figure 2. The system can accept requests by both a dedicated client and by generic user agents as DAML+OIL descriptions.

The client is a Java applet (an extremely light client, in view of application on devices such as PDAs) that sends, via SOAP (http://www.w3. org/TR/SOAP/), an advertisement i.e., a description of the request to be matched, as a string in KRSS (Knowledge Representation System Specification) (http://www.bell-labs.com/ user/pfps/papers/krss-spec.ps) syntax, Figure 3 shows the content for an example advertisement in KRSS.
Example Demand: (and APARTMENT (all HASROOMS BEDROOM) (at-most 0 HASPETS))

POST /soap/servlet/rpcrouter HTTP/1.0 Host: localhost: 8070 Content-Type: text/xml Content-Length: 597 SOAPAction: "" <SOAP-ENV:Envelope

xmlns : SOAP-ENV="http://schemas. $x m l$ soap.org/soap/envelope/" xmlns:xsi="http://www.w3.org/1999/xMLSchema-instance" xmlns:xsd="http://www.w3.org/1999/XMLSchema"〉〈SOAP-ENV : Body> <ns1:translate xmlns:ns1="urn: demo1:Translator"

SOAP-ENV : encodingStyle="http://schemas. xml soap.org/soap/encoding/"> <text xsi:type="xsd:string"> (and APARTMENT (all HASROOMS BEDROOM) (at-most 0 HASPETS)) $</$ text $>\langle$ uri

xsi:type="xsd:string">http://www. example.org/rent-toy-ontology\#</uri> $\langle$ requestType xsi:type="xsd:string">demand $\langle/$ requestType $\rangle$

$\langle/$ ns1:translate $\rangle /$ SOAP-ENV:Body> </SOAP-ENV:Envelope>

Fig. 3. An example KRSS SOAP packet.

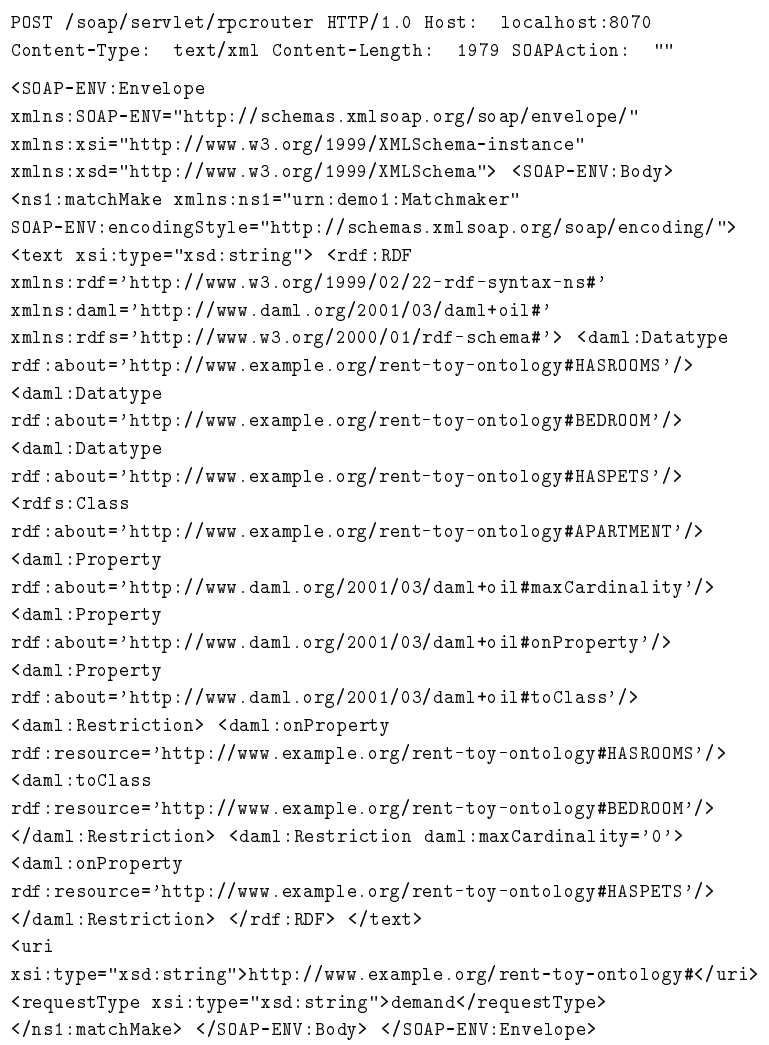

Fig. 4. The DAML translation of the previous KRSS description.

The SOAP packet contains the string and the URI of the reference ontology. The communication module is a web service whose main purpose is the translation of KRSS descriptions into portable DAML+OIL ones. The module, upon receipt of the packet, transforms the string in a DAML+OIL formatted description, using Jena APIs (http://www.hpl.hp.com/semweb/). Figure 4 shows the corresponding packet.

The description is forwarded to the Matchmaking service, which is the principal module of the architecture. It receives the SOAP packet, extracts the code and the URI that references 
the ontology requested. The matchmaking engine preliminarily checks for satisfiability w.r.t. the referenced ontology. If the check succeeds it carries out the matchmaking process with all descriptions in the repository corresponding to the given ontology, as will be described in detail in the next subsection.

The system accepts two types of requests, advertisement and query. For the first one, the system will store the request. In this way, satisfiable queries/demands that remain unmatched will be automatically reexamined when new supplies are provided, and notification will be provided for successful match. The same service is available for unmatched supplies. The query service does not permanently store demand/supply descriptions.

The matchmaker output is forwarded according to specifications included int the SOAP packet and can be formatted as an e-mail, a SMS (Small Message System) or a JSP (Java Servlet Page), if the request has been submitted by the client. If the request has been submitted by an agent, the response will be sent to the communication service for translation from KRSS to DAML+OIL.

Potential and partial matches can also trigger further communication services. For partial matches, on the basis of the matchmaker response, a demand can be revised, e.g., relaxing some constraint. For potential matches the system can ask the supplier of the advertisement whether some features, although not advertised, are available anyway.

It should be noticed that the architecture can simply be modified to host other reasoners, such as FaCT or Racer. The rationale of the choice of the CLASSIC system, apart from the obviously useful availability of concrete datatypes and the possibility to extend its functionalities through test functions, is that its polynomial time inference allows practically synchronous operations, even with large ontologies. Recently, DAML-S [24] has been proposed as a standard for the automation of web services and processes. It is a set of markup constructs based on DAML+OIL and we plan to implement support for it in the version of the system, where we will introduce also a negotiation service, which is currently not supported.

\subsection{The matching engine}

Our matching engine, whose architecture is shown in Figure 1, is based on Java servlets; it embeds the NeoClassic reasoner and communicates with the reasoner running as a background daemon. At this stage of the work, the system is not fully transactional, so requests have to be serialized by the engine.

The system receives the KRSS string describing the demand/supply and the URI referencing the proper ontology. The reasoner checks the description for consistency; if it fails, based on the reasoner output, the system provides an error message stating the error has occurred. Otherwise, the proper matchmaking process takes place. The NeoClassic standard [4] subsumption algorithm has been adapted in accordance with the matchmaking algorithms presented in section [4]. Each match can return a 0, which means exact match or a value $>0$. Recall that returned values for partial matches and potential matches have logically different meaning and matching descriptions are sorted in different sets. The matching engine may then return up to three separate result sets.

The matchmaker can also use weights to increase the relevance of concepts. The weight is a positive integer that takes into account the occurrence of a role or a concept w.r.t. all Demands and Supplies available for a reference ontology. For each concept or role present in an advertisement, in normal form, that is $A \in D_{\text {names+ }}$ or a concept $(\geq x R) \in D_{\sharp}$ or $(\leq x R) \in D_{\sharp}$, the corresponding weight is increased after each matching process. Obviously, the matchmaking algorithm is modified in that increments are no more unitary but correspond to the assigned weights.

It is noteworthy that the ranking also prevents advertisements from being submitted in an extremely generic way. Simple subsumption matching [26] without ranking, in fact, favors unfair generic advertisements, which will be present in practically any retrieved set. Instead, in our system, though logically potentially matching, generic supplies are given a high rank, which penalizes them. 


\section{Conclusion}

We have addressed the problem of matchmaking of web services from a knowledge representation perspective. We proposed match categorization in terms of exact match, potential match and partial match and rank of matches within categories. In accordance with the above properties we have devised algorithms for matchmaking. The approach has been implemented in a matchmaking facilitator. The core engine has been implemented adapting the NeoClassic reasoner, and we have shown that, although with a reduced expressiveness w.r.t. more recent reasoners, CLASSIC can be effectively used for this class of problems.

\section{References}

[1] T. BERNERS-LEE AND J. HENDLER AND O. LASSILA, The Semantic Web, Scientific American, may, 2001, available at: http://www.w3.org/2001/sw/

[2] A. BorgidA, Description Logics in Data Management, IEEE Transactions on Knowledge and Data Engineering, vol. 7, no. 5, pp. 671-682, 1995.

[3] A. BORGIDA AND R. J. BRACHMAN AND D. L. MCGUINNESS AND A. RESNICK, LORI, CLASSIC: A Structural Data Model for Objects, Proceedings of the ACM SIGMOD International Conference on Management of Data, pp. 59-67, 1989.

[4] Alexander BorgidA AND Peter F. PATElSCHNEIDER, A Semantics and Complete Algorithm for Subsumption in the CLASSIC Description Logic, Journal of Artificial Intelligence Research, vol. 1, pp. 277-308, Morgan Kaufmann, Los Altos, 1994.

[5] D. Calvanese, Reasoning with Inclusion Axioms in Description Logics: Algorithms and Complexity, Proceedings of the Twelfth European Conference on Artificial Intelligence (ECAI'96), pp. 303-307, John Wiley \& Sons, 1996.

[6] D. Calvanese, G. De Giacomo and M. LenZerini, On the Decidability of Query Containment under Constraints, Proceedings of the Seventeenth ACM SIGACT SIGMOD SIGART Symposium on Principles of Database Systems (PODS'98), pp. 149-158, 1998.

[7] F. CASATi AND M. C. SHAn, Dynamic and Adaptive Composition of E-Services, Information Systems, vol. 26, pp. 143-163, 2001.

[8] E. Christensen, F. Curbera, G. Meredith And S. WEERAWARANA, Web Services Description Language, 2001, available at: http: //www.w3.org/ TR/wsdl,
[9] E. Di SCIASCIO, F.M. DONINI, M. MongIELlo AND G. Piscitelli, A Knowledge-Based System for Person-to-Person E-Commerce, Proc. Workshop on Applications of Description Logics (KI 2001), 2001.

[10] F. M. DONINI, M. LENZERINI, D. NARDI AND A SCHAERF, Reasoning in Description Logics, Principles of Knowledge Representation, Gerhard Brewka, pp. 93-238, CSLI Publications, Studies in Logic, Language and Information, 1996.

[11] D. FENSEl, F. VAN HARMELEN, I. HORROCKS, D. MCGUINNESS AND P. F. PATEL-SCHNEIDER, OIL: An Ontology Infrastructure for the Semantic Web, IEEE Intelligent Systems, vol. 16, no. 2, pp. 38-45, 2001, available at: http: //www . computer.org/intelligent/ ex2001/x2toc.htm

[12] T. FININ AND R. FRITZSON AND D. MCKAY AND R. MCENTIRE, KQML as an Agent Communication Language, Proceedings of the Third International Conference on Information and Knowledge Management (CIKM'94), Gaitherburg, pp. 456-463, ACM, New York, 1994.

[13] Y. GIL AND S. RAMACHANDRAN, PHOSPHORUS: a Task based Agent Matchmaker, Proc. AGENTS '01, Montreal, pp. 110-111, ACM, New York, 2001.

[14] J. GonZales-CASTILlo AND D. TRAstour AND C. BARTOLINI, Description Logics for Matchmaking of Services, Proc. Workshop on Applications of Description Logics (KI 2001), September, 2001.

[15] V. HaARsLeV AND R. Moller, RACER System Description, International Joint Conference on Automated Reasoning, IJCAR'2001, pp. 701-705, Springer-Verlag, Berlin, 2001.

[16] Y. HofFner, C. FACCIORUSSO, S. FIELD AND A. SCHADE, Distribution Issues in the Design and Implementation of a Virtual Market Place, Computer Networks, vol. 32, pp. 717-730, 2000.

[17] I. Horrocks, The FaCT System, Automated Reasoning with Analytic Tableaux and Related Methods: International Conference Tableaux'98, may, Lecture Notes in Artificial Intelligence, 1397, pp. 307-312, Springer-Verlag, Berlin, 1998.

[18] I. HORROCKS AND ET AL., DAML+OIL language specification, 2001, available at: http: //www . daml.org/2001/03/daml+oil-index

[19] N. KARACAPILIDIS AND P. MORAITIS, Building an Agent-Mediated Electronic Commerce System with Decision Analysis Features, Decision Support Systems, vol. 32, pp. 53-69, 2001.

[20] D. KUOKKA AND L. HARADA, Integrating Information Via Matchmaking, Journal of Intelligent Information Systems, vol. 6, pp. 261-279, 1996.

[21] O. LASSILA AND R. R. SwICK, Resource Description Framework, 1999, available at: http: //www . w3.org/TR/REC-rdf-syntaxl

[22] B. NEBEL, Terminological Reasoning is Inherently Intractable, Artificial Intelligence, vol. 43, pp. 235249, 1990. 
[23] T. Di NoIA, E. Di ScIascio, F.M. Donini AND M. MoNGIELLO, Semantic Matchmaking in a P-2-P Electronic Marketplace, ACM SAC-03, Melbourne, Florida, USA, pp. 582-586, 2003.

[24] M. PaOlucci, T. KaWAmura, T.R. Payne and K. SYCARA, Semantic Matching of Web Services Capabilities, The Semantic Web - ISWC 2002, Lecture Notes in Computer Science, 2342, pp. 333-347, Springer-Verlag, 2002.

[25] K. Sycara, S. Widoff, M. Klusch and J. LU, LARKS: Dynamic Matchmaking Among Heterogeneus Ssoftware Agents in Cyberspace, $\mathrm{Au}$ tonomous agents and multi-agent systems, vol. 5, pp. 173-203, 2002.

[26] D. TRAstour AND C. BARtolini AND C. PRIEST, Proc. $W W W$ '02, Semantic Web Support for the Business-to-Business E-Commerce Lifecycle, Honolulu, pp. 89-98, ACM, New York, 2002.

[27] H. WANG, S. LIAO AND L. LIAO, Modeling Constraint-Based Negotiating Agents, Decision Support Systems, vol. 33, pp. 201-217, 2002.

Received: June, 2003

Accepted: September, 2003

Contact address:

S. Colucci, T. Di Noia, E. Di Sciascio, M. Mongiello Politecnico di Bar

Via Re David, 200

70125 Bari, Italy e-mail:

$\{$ s.colucci,t.dinoia, disciascio,mongiello\}@poliba.it

F. M. Donini

Universitá della Tuscia

Via S. Carlo 32

01100 Viterbo, Italy

e-mail: donini@unitus.it

FRANCESCO M. DONINI received the Master's degree in electronics engineering from the University of Rome "La Sapienza" in 1988. He received the Ph.D. in computer science from the same university in 1992. From 1991 to 1998 he was researcher/assistant professor at the Department of Computer and System Science of the University of Rome "La Sapienza". Since 1998 he is an associate professor, first at the Technical University of Bari, and currently at the University of Tuscia in Viterbo. He is the coauthor of many papers in international journals, as well as in the proceedings of international conferences. The paper "Tractable concept languages", presented at the conference IJCAI-91 (1991), received the best paper award. He is responsible for local university research projects since 1996, and for CNR research projects. He is the editor of the area "Concept-Based Knowledge Representation" for the journal ETAI - electronic transactions on artificial intelligence - published by the Royal Swedish Academy.

MARINA MongIELLO received the laurea "cum laude" degree in computer science from the University of Bari in 1993 and the Ph.D. degree in 2001 from the University of Catania. In 2002 she joined the Technical University of Bari as an assistant professor. Her research interests include multimedia information retrieval, knowledge-based systems for e-commerce and model checking.

SIMONA COLUCCI received the laurea degree "cum laude" in management engineering from the Technical University of Bari in 2002. Currently she is a Ph.D. student in information technology engineering at the Technical University of Bari. Her research interests include description logics and their application to e-commerce and skills management. On these topics she has published papers in journals and proceedings of international conferences.

TOMMASO DI NOIA received the laurea degree in electronic engineering from the Technical University of Bari in 2002. Currently he is a Ph.D. student in information technology engineering at the Technical University of Bari. His research interests include description logics and their application to e-commerce issues and knowledge management. On these topics he has published papers in journals and proceedings of international conferences.

EUGENIO Di SCIASCIO received the laurea "cum laude" degree in electronic engineering from the University of Bari in 1989 and the Ph.D. degree in 1994 from the Technical University of Bari. In 1992 he joined the University of Lecce as an assistant professor. He is currently an associate professor of information technology engineering at the Technical University of Bari, where he is responsible for various research projects. His research interests include knowledge-based systems for e-commerce, image and video processing and multimedia information retrieval. On these topics he has authored or co-authored over eighty paper in international journals and proceedings of international conferences. 